\title{
A RF Broadband Biasing Holder for Ultrafast Stroboscopic Electron Microscopy
}

Karl B. Schliep ${ }^{1}$, Michael B. Katz ${ }^{1}$, and June W. Lau ${ }^{1}$

1. The National Institute of Standards and Technology, Material Measurement Laboratory, Gaithersburg, USA

Ultrafast electron microscopy has considerably advanced our understanding of laser-driven phenomena across a wide range of fields [1,2]. These phenomena are observed using a stroboscopic pump-probe process where a reversible specimen processes is initiated (pumped) using some in situ trigger and then imaged (probed) by a temporally short electron packet. In most ultrafast electron microscopes the pump and probe pulses are generated by femtosecond pulsed lasers in the extraction of photoelectron packets and the photoexcitation of the specimen; however, with the invention of laser-free pulsed electron sources [3], a new mechanism for exciting specimen dynamics is needed to replace the lack of laser in the system. Here we present a design for an in situ transmission electron microscope holder that can deliver highfidelity, low-loss broadband alternating electrical signals to the sample in the microwave frequency range. This holder enables electrical processes to be directly stimulated thus mitigating the operational frequency and resolution limitations inherent to laser-induced specimen excitation. This holder design was based on a standard commercially available electrical biasing feedthrough holder and a carrier system. Although standard biasing holders can efficiently transmit DC and low AC signals to the sample, as the frequency increases transmission losses and reflections dominate the signal propagation. To overcome these issues, the carrier properties can be designed to mitigate losses inherent to high-frequency signal transmission while staying within simple manufacturing constraints.

Our design assumes the signal can be effectively transmitted from the microwave source through standard connections to the holder and then inside the microscope via feedthroughs. The feedthrough cables terminate at a commutable specimen carrier that is designed to transmit the signal from the holder output to the TEM specimen. The carrier relies on coplanar waveguides (CPW) to transmit the signal to the sample and can, therefore, be adapted to minimize the losses by varying the CPW dimensions and design. Multiple traces and grounds are used to optimize the available space of the holder and allow for a broadrange of inputs and outputs for in situ signal transmission and detection. A variety of material properties and spatial dimensions were considered in the design of the carrier. The basic design of the specimen carrier is shown in Figure 1.

The carrier design was investigated using the RF module of COMSOL. To simulate the carrier, a single CPW structure was used to alleviate computation time and to isolate the effect of varying dimensions on the scattering parameters. The simulations revealed that by varying the physical dimensions of the CPW its transmission properties across a range of frequencies changed significantly, see Figure 2. This work highlights the fact that no one carrier design will satisfy all frequencies and that different carrier will be necessary for every frequency band of interest. Understanding the losses along the sample carrier will allow for a more accurate estimation of the amount of signal that reaches the specimen and can greatly aid in the interpretation of ultrafast results, which are often quite subtle. Further work on this topic will add in the effects of a constant magnetic field to simulate being in a TEM, the perpendicular signal launch at the connection between the feedthroughs and the carrier, and the response of nanoelectromechanical TEM samples [4]. 
References:

[1] Adhikari, A., et al., ACS Appl. Mater. Interfaces 9 (2017), p. 3.

[2] Shorokhov, D. and Zewail, A. H., J. Chem. Phys. 144 (2016), p. 080901.

[3] Qiu, J., et al., Ultramicroscopy 161 (2016), p. 130.

[4] This research was performed while the author held an NRC Research Associateship award at the National Institute of Standards and Technology in Gaithersburg, MD

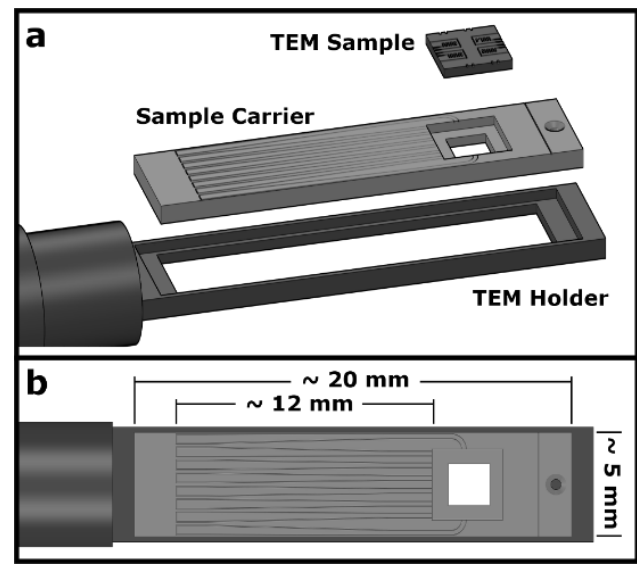

Figure 1. Schematic of the sample holder, carrier, and TEM sample. a) How the pieces fit together. b) Typical dimensions of the carrier and a nominal design configured with 8 traces arranged such that the TEM sample can be rotated to provide two separate orientation. This allows the sample to be made for 16 total electrical connections, 8 per experiment.

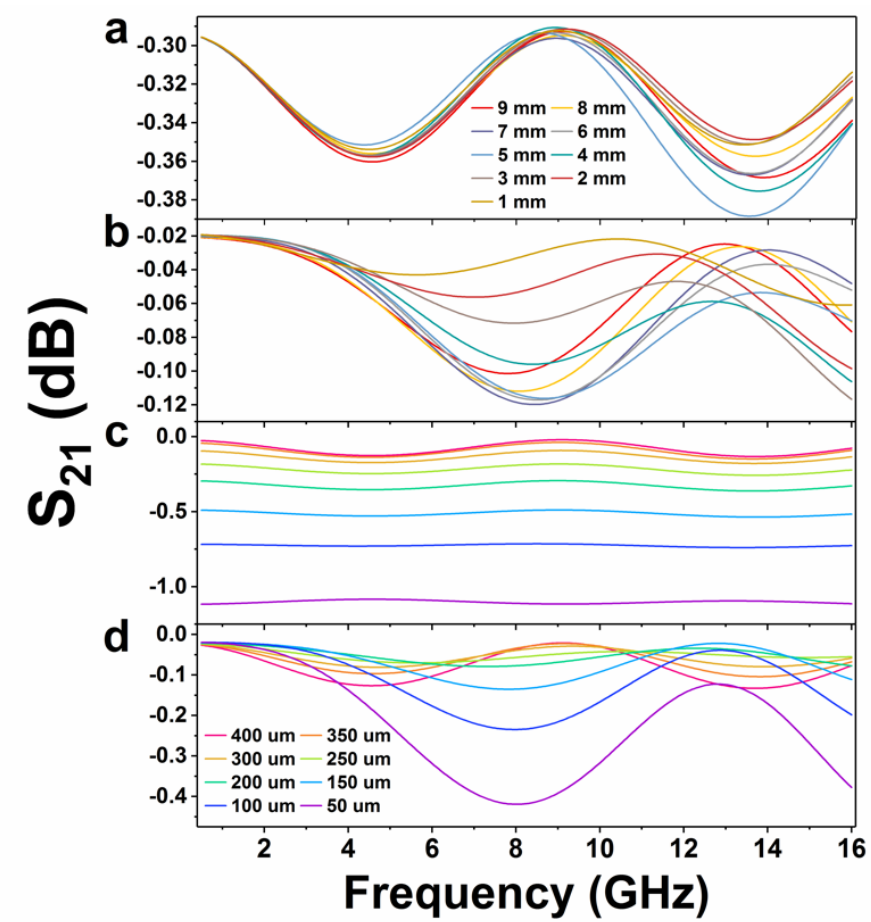

Figure 2. COMSOL simulation results on the transmission of signal for different designs. Changing waveguide length for a tapered conductor width from $400 \mu \mathrm{m}$ to $200 \mu \mathrm{m}$ with a) a constant impedance of $50 \mathrm{ohms}$ and b) a constant gap width of $50 \mu \mathrm{m}$. Changing waveguide taper width at a constant length of $10 \mathrm{~mm}$ for c) constant impedance of $50 \mathrm{ohms}$ and d) constant gap width of $50 \mu \mathrm{m}$. 